\title{
Neues zu Leben und Werk von Leonhart Fuchs aus seinen Briefen an Joachim Camerarius I. und II. in der Trew-Sammlung*
}

\author{
Von Gerhard Fightner, Tübingen
}

Man hat Leonhart Fuchs im 16. Jahrhundert als neuen Galen und als neuen Dioskurides gefeiert ${ }^{1}$, in Anspielung auf sein medizinisches wie auf sein botanisches Werk. Wenn das auch panegyrischer Stil der Zeit ist, so lohnt es sich doch zu fragen, woher die Faszination rührte, die von Fuchs ausging. Eberhard Stübler hat mit seiner verdienstvollen Fuchs-Monographie $^{2}$ gezeigt, daß Fuchs den wissenschaftlichen Bestrebungen und Gedanken seiner Zeit einen besonders klar formulierten Ausdruck zu geben vermochte und daß zum Verständnis des Fuchsschen Werkes die medizinischen Arbeiten ebenso zu beachten sind wie die botanischen.

Wir möchten hinzufügen: auch die Korrespondenz, die Fuchs führte, ist in ihrer Unmittelbarkeit und Spontaneität eine Quelle ersten Ranges, um Person und Werk zu erfassen.

Freilich ist es gegenüber der Fülle des gedruckten Werkes nicht eben viel, was von Fuchsens eigener Hand erhalten und bekannt ist ${ }^{3}$. Um so merkwürdiger erscheint es, daß die Briefe bisher kaum beachtet, geschweige denn ausgeschöpft wurden, die Fuchs an Joachim Camerarius d. ̈̈. (1500

* Vortrag, gehalten am 4. Januar 1968 beim V. Symposium der Gesellschaft für Wissenschaftsgeschichte e.V. in Düsseldorf.

1 Nicolaus ReUSNeR: Icones sive imagines virorum literis illustrium quorum fide et doctrina religionis et bonarum literarum studia, nostra patrumque memoria, in Germania praesertim, in integrum sunt restituta. [Etc.] Argentorati 1587, f. T[r] :

Artem si medicam spectas, nouus ipse Galenus;

Si plantas, nouus en exsto Dioscorides.

2 Eberhard Stübler, Leonhart Fuchs - Leben und Werk, München 1928 (= Münchner Beiträge zur Geschichte und Literatur der Naturwissenschaften und Medizin 13/14).

3 Neben dem gewaltigen Kräuterbuchmanuskript (vgl. dazu KURT Ganzinger, Ein Kräuterbuchmanuskript des Leonhart Fuchs in der Wiener Nationalbibliothek, in Sudhoffs Arch. 43 (1959) 212-224) sind nur wenige Schriftstücke von Fuchs im Tübinger Universitäts-Archiv (im folgenden abgekürzt als UAT) und im Hauptstaatsarchiv Stuttgart (abgekürzt HStASt) bekannt, darunter ein Briefwechsel mit Herzog Christoph wegen der Besetzung der Leibarztstelle aus dem Jahre 1550 (HStASt A 20 B. 43). 
bis 1574) und Joachim Camerarius d. J. (1534-1598) ${ }^{4}$ geschrieben hat, jene Briefe, die lange Zeit in den Händen der Nachkommen von Camerarius waren und spätestens im Jahr 1750 von Christoph JAGOB Trew für seine berühmte Briefsammlung ${ }^{5}$ erworben wurden. Denn Casimir Christoph Schmi(E) DEL, der im Auftrage Trews 1754 die nachgelassenen botanischen Werke Konrad Ges(s)ners herausgab ${ }^{6}$, druckte in einer vorangestellten Vita Ges(s)ners auch einige kleine Bruchstücke dieser Briefe von Fuchs ab, die sich auf die Neubearbeitung seiner Historia stirpium bezogen, und machte damit die Öffentlichkeit mit der Existenz dieser Briefe bekannt.

Nach Trews Tod kam seine Sammlung an die Universität Altdorf und nach deren Auflösung in die Universitätsbibliothek von Erlangen, wo sich die Briefe noch heute befinden.

In neuerer Zeit sind die Briefe, soweit wir sehen, nur von Hermann Jordan $^{7}$ und Eberhard Stübler ${ }^{8}$ beachtet worden, die von 12 bzw. 11 Briefen von Fuchs sprechen. Stübler hat einen der Briefe (teilweise in Faksimile) veröffentlicht ${ }^{9}$, scheint aber von den anderen Briefen keine eingehende Notiz genommen zu haben.

${ }^{4} \mathrm{Zu}$ Joachim Camerarius d. Ä. vgl. Neue Deutsche Biographie, Band 3 (1957), S. 104f. (Friedrich StäHLIN). Dort weitere Literatur. Zu Joachim Camerarius d. J. vgl. Biographisches Lexikon der hervorragenden Ärzte, herausgegeben von A. HirscH, Band 1, (3. Auflage 1962) S. 808, und Nürnbergisches Gelehrten-Lexicon (G. A. WILL und Cн. C. Nopitsch) T. 1, (1755) S. 173, und T. 5, (1802) S. 145.

5 Vgl. dazu El. Schmidt-Herrling, Die Briefsammlung des Nürnberger Arztes Christoph Jacob Trew (1695-1769) in der Universitätsbibliothek Erlangen, Erlangen 1940 (= Katalog der Handschriften der Universitätsbibliothek Erlangen, Band 5), wo in der Einleitung, S. XIX ff., die Geschichte der Camerarius-Briefsammlung eingehend geschildert wird.

${ }^{6}$ Conradi Gesneri ... Opera botanica ... edidit et praefatus est D. Casimirus Christophorus Schmiedel, T. 1.2, Norimbergae 1754. 1771.

7 Hermann Jordan, Reformation und gelehrte Bildung in der Markgrafschaft AnsbachBayreuth, 1. Teil, Leipzig 1917 (= Quellen und Forschungen zur bayerischen Kirchengeschichte I, 1) S. 206, A. 2.

8 Stübler (1928) S. 9 (173), A. 2.

9 StüBler (1928), Abb. nach S. 16 (180). Leider enthält die Transkription auf S. 10 (174) etliche sinnentstellende Fehler, z.B. 'schola nova' statt 'schola nostra' (Zeile 12), 'caro (?) viro' statt 'hac vice' (Zeile 22) und 'familia, quam semper Deus incolumem esse servet' statt 'familia, quam precor Deus incolumem conservet'. An einer Stelle (S. 9 [173]) konstruiert St. aus einem Lesefehler (S. 10 (174), Zeile 19 des Briefes) 'honorifico' statt 'honorifice' den Beweis für Schwierigkeiten, die Fuchs mit Herzog Ulrich gehabt habe. Der ganze Satz lautet: Quid honorifice de [me] loquatur ex aliis forte cognosces. Das Subjekt des Satzes ist noch Caspar Volland, sein Kollege, den er im Vorsatz eine pestis literarum genannt hat. Aber nach dem 'de', das sich nicht auf ein 
Der Katalog der Trew-Sammlung verzeichnet 27 Briefe $^{10}: 26$ an Joachim Camerarius d. A. und einen an Joachim Camerarius d.J. In Wirklichkeit sind es an Joachim Camerarius d. Ä. nur 25 Briefe, denn bei Brief 10 handelt es sich, wie aus der fehlenden Anrede und dem Schluß (Vale ut in literis) hervorgeht, lediglich um ein Postskript, vermutlich zu Brief $9{ }^{11}$.

Die sämtlich lateinisch geschriebenen Briefe stammen aus den Jahren 1542 (1541 ?)-1565, also aus der Tübinger Zeit von Fuchs, in der er auf der Höhe des Ruhmes und der Anerkennung stand, in der ihn aber auch ein lange währender Kleinkrieg mit dem württembergischen Hof sowie die politischen und religiösen Unruhen, die Deutschland betrafen, niederdrückten und betroffen machten. Denn das ist der erste starke Eindruck aus diesen Briefen: hier spricht kein Stubengelehrter, den man hinter einem so umfänglichen literarischen Werk, das sich um die richtige Exegese alter Traditionen bemüht, vermuten könnte, sondern ein Mann, der wachen Anteil am Geschehen der Zeit nimmt und besonders ins Reformationsgeschehen auch aktiv, ratend und Stellung nehmend, eingreift. Wenn es richtig ist, daß die humanistische wissenschaftliche Diskussion Fuchs den Zugang zum reformatorischen Gedankengut erleichterte, so wäre umgekehrt übrigens zu fragen, od nicht auch reformatorische Prinzipien ihren Niederschlag im wissenschaftlichen Werk von Fuchs gefunden haben. Das ist eine noch ungeklärte Frage, doch zeigen uns diese Briefe von Fuchs zumindest, wie eng sein Leben mit der Reformation verflochten war.

Vergegenwärtigen wir uns die biographische Situation zu Beginn des Briefwechsels mit Joachim Camerarius d. Ä.

Fuchs hatte sich schon früh einen Namen gemacht durch seinen heftigen Kampf gegen die arabistischen Ärzte und war als ein weithin bekannter Mann vom heimgekehrten Herzog Ulrich 1535 zusammen mit Camerarius an die Universität Tübingen berufen worden. Sowohl Fuchs wie Camerarius wurden gerühmt wegen ihrer hervorragenden Kenntnis der klassischen Sprachen, und es ist bezeichnend, daß man sich von solchen Männern vorzüglich die Erneuerung der Universität versprach. Camerarius, der Griechisch und Latein zu lehren hatte, galt freilich als der noch Berühmtere:

'honorifico' zurückbeziehen könnte, hat Fuchs das Bezugswort vergessen. Wir möchten 'me' ergänzen.

10 El. Schmidt-Herrling (1940), S. $195 \mathrm{f}$.

11 Trotzdem soll die von E. Schmidt-Herrling eingeführte Zählung beibehalten werden, um die Orientierung zu erleichtern und Mißverständnisse zu vermeiden. 
das drückte sich im Gehalt aus ${ }^{12}$. Man kann sich gut vorstellen, wie Fuchs und Camerarius in dieser ersten Tübinger Zeit freundschaftlichen Kontakt zueinander fanden und ihre Gedanken und Pläne miteinander besprachen, zumal beide weit über ihr Fachgebiet hinaus interessiert waren (Camerarius galt seinerzeit auch als bedeutender Mathematiker). Obwohl Camerarius die in ihn gesetzten Hoffnungen nicht enttäuscht haben dürfte, haben sich. wohl bald Differenzen mit dem Hof ergeben, so daß Camerarius Ende 1541 Tübingen verließ und später einem Ruf nach Leipzig folgte ${ }^{13}$.

$\mathrm{Zu}$ dieser Zeit beginnt der Briefwechsel. Freilich läßt sich gerade der erste der erhaltenen Briefe, der undatiert ist, nicht ganz präzis einordnen. Der Inhalt - es handelt sich um eine Diskussion über die Arten der Anthemis, die inhaltlich und zum Teil wörtlich genauso in der Historia stirpium von 1542 erscheint $^{14}$ - der Inhalt also spricht dafür, daß dieser Brief noch vor dem Erscheinen der Historia stirpium, deren Vorwort auf den 1. März 1542 datiert ist, geschrieben wurde. An diesem Brief ist aber auch der Anfang abgeschnitten worden, wie einige stehengebliebene Unterlängen zeigen. Das hat vermutlich Camerarius selbst getan, der sicherlich früh damit begonnen hat, Briefe zur Veröffentlichung zu sammeln und diesen Briefanfang wohl als nicht geeignet für die Öffentlichkeit ansah. Es ist verlokkend anzunehmen, daß Fuchs hier auf die Gründe für den Weggang von Camerarius eingegangen ist. Camerarius hat sich offensichtlich ängstlich bemüht, nichts von diesen Gründen an die Öffentlichkeit dringen zu lassen.

Die übrigen Briefe lassen sich, auch wenn die Jahreszahl im Datum fehlt, aufgrund inhaltlicher Kriterien genau datieren. Leider scheinen sich die Antwortbriefe von Camerarius ebenso wie der eine Brief von Joachim Camerarius d.J., auf den Fuchs antwortet, nicht erhalten zu haben. Es finden sich lediglich zwei zu diesem Briefwechsel gehörige Briefe von Camerarius an Fuchs in der 1583 gedruckten Briefsammlung ${ }^{15}$, die von den Söhnen von

12 Camerarius bekam von Anfang an 200 Gulden pro Jahr, Fuchs zunächst nur 160 Gulden pro Jahr. Vgl. UAT 6/7a, f. 20 r.

13 Vgl. dazu [Rudolf Rотн], Urkunden zur Geschichte der Universität Tübingen aus den Jahren 1476 bis 1550, Tübingen 1877, S. 425-428.

${ }^{14}$ Leonhart Fuchs, De historia stirpium commentarii insignes, [etc], Basileae 1542. Cap. VIII.

15 Joachim Camerarius, Epistolarum familiarium libri VI. Nunc primum post ipsius obitum singulari studio a filiis editi, Francofurti 1583, p. 338-340. Bei dem ersten Brief handelt es sich um die Antwort auf den Fuchs-Brief Nr. 19 vom 5. September 1553, beim zweiten um die Antwort auf den Fuchs-Brief Nr. 16 vom 3. September 1551. 
Camerarius herausgegeben wurde. Auch von Fuchs sind nicht alle Briefe an Camerarius erhalten. Es fällt aber auf, daß in den ersten Jahren nach dem Weggang von Camerarius häufiger Briefe gewechselt wurden. Allerdings war Camerarius in den fünfziger Jahren nachweislich mehrmals in Tübingen zu Besuch ${ }^{16}$. Doch scheint sich auch das freundschaftliche Verhältnis etwas abgekühlt zu haben, vielleicht nicht zuletzt durch die unterschiedliche Beurteilung des Osiandrischen Streits.

Diese Briefe von Fuchs vermitteln uns einerseits eine wertvolle Selbstcharakteristik, sie beschwören die Atmosphäre von Wirkungskreis und -zeit, andererseits geben sie uns eine Fülle von Einzelnachrichten zu seinem Leben, dessen Verflochtensein mit Reformations- und Zeitgeschichte sowie zu seinem wissenschaftlichen Werk. Davon kann hier nur einiges Charakteristische (geordnet nach den genannten Stichworten) geboten werden.

Die Stetigkeit, die man im Leben von Fuchs darin sehen könnte, daß er seit seiner Berufung bis ans Lebensende in Tübingen blieb, ist scheinbar. In Wirklichkeit hat er früh von Tübingen fortgestrebt, wo er sich aus mancherlei Gründen nicht wohl fühlte. Das zeigt bereits der Briefwechsel mit Herzog Albrecht von Preußen in den Jahren 1537/38, als dieser ihn vergeblich als Leibarzt für den König von Dänemark zu gewinnen suchte, Fuchs sich aber nicht abgeneigt zeigte, in die Dienste von Markgraf Albrecht zu treten ${ }^{17}$. Vollends deutlich wird es bei der Berufung nach Pisa durch Herzog Cosimo Medici von Florenz im Jahre 1544. "In welchen Zwiespalt mich die Berufung nach Italien versetzt hat und wie sie alle meine Pläne durcheinander gebracht hat, kannst du dir leicht vorstellen » schrieb Fuchs damals an Camerarius (Brief 8 vom 1. November 1544). Er ist zwar entschlossen, den Ruf, der ehrenvoll und in materieller Hinsicht höchst verlockend war, nicht anzunehmen, weil er Gefahren wittert und vor allem des religiösen Bekenntnisses halber. «Wenn aber mein Sinn auch nicht auf Italien gerichtet ist, so heißt das doch nicht, daß ich deswegen hierzubleiben gedenke. Es drängt mich vieles, mir wegen einer anderen Stellung Gedanken zu machen, vor allem die große Schar meiner Kinder, für die ich unbedingt besser vorsorgen muß.» Am liebsten wäre er in Leipzig neben Camerarius, aber auch nach Augsburg würde er bei ehrenvollen Bedingungen gehen, obwohl ihm das Lehramt an einer richtigen Universität

16 Vgl. die Eintragungen in den Jahresrechnungen des Supremus Deputatus UAT 6/7c, f. $23 \mathrm{r}(1550 / 51)$ und f. $114 \mathrm{r}(1553 / 54), 6 / 8$, f. $57 \mathrm{v}(1559 / 60)$.

17 Johannes Vorgt, Briefwechsel der berühmtesten Gelehrten des Zeitalters der Reformation mit Herzog Albrecht von Preußen, Königsberg 1841, besonders S. 262-270. 
lieber wäre. Denn er sehe, daß er zum Lehren geboren sei ${ }^{18}$. Im Jahre 1548 fragt er nochmals bei Camerarius an, ob er ihm nicht eine Stellung in Sachsen vermitteln könne (Brief 12).

Welche Gründe hatte Fuchs für sein Fortstreben?

Einmal fühlte er sich in Tübingen, seit Camerarius nicht mehr da war, allein, ohne Freunde und Gesinnungsgenossen ${ }^{19}$. Das dürfte besonders für den kleinen Kreis der eigenen Fakultät gegolten haben. Der Kollege Michael Rucker war bereits im Jahre 1530 - einige Jahre vor der Universitätsreform - berufen worden ${ }^{20}$, $\mathrm{kam}$ also aus der alten Universitätstradition und war auch sonst eher ein konservativer Typ: er verschloß sich der Reformation ${ }^{21}$. Und auch seine wissenschaftliche und pädagogische Leidenschaft war nicht so groß, daß er dadurch Fuchs nähergekommen wäre: wir kennen keine Buchveröffentlichung von Rucker, und es vergeht kaum ein Semester, ohne daß wir ihn unter denen finden, die Strafe für versäumte Lektionen zu zahlen haben ${ }^{22}$. Fuchs erwähnt denn auch nur seinen Tod im Jahre $1561^{23}$.

Gegen den Willen der Universität und sicherlich gegen den Willen von Fuchs hatte im Jahre 1540 auf Wunsch Herzog Ulrichs dessen Leibarzt Wolfgang Thalhäuser die medizinische Professur erhalten ${ }^{24}$. Die Universität hatte betont, man benötige keinen dritten Lehrstuhl, und im übrigen sei er schon Sebald Hawenreut $(t)$ er versprochen worden ${ }^{25}$. Hawenreut $(t) e r$,

18 Alle Zitate aus Brief 8. Vgl. für das letzte auch die Bemerkung in Brief 12 vom 13.Dezember 1548: «Habeo frequens auditorium, viginti enim quator sunt qui me docentem medica quotidie audiunt.» Sie wirft ein bemerkenswertes Licht auf die pädagogische Kunst und Leidenschaft von Fuchs.

19 «Accedit quod post tuum a nobis discessum nullum habuerim amicum fidum, cum quo de rebus meis tuto conferre potuerim» (Brief 8 ).

$20 \mathrm{Vg}$. UAT $5 / 13$, f. $60 \mathrm{v}$.

21 Vgl. [Rudolf Roth], Urkunden zur Geschichte der Universität Tübingen aus den Jahren 1476 bis 1550, Tübingen 1877, S. 166.

22 Vgl. die Eintragungen in den Jahresrechnungen unter «neglectae lectiones», UAT $6 / 7 \mathrm{a}-\mathrm{c}$ und 8.

23 «D. Michael proximo autumno [8. Oktober 1561] apoplexia correptus interiit» (Brief 23 vom 21. Dezember 1561).

24 Vgl. den Beginn der Besoldung in den Jahresrechnungen «ad angariam Luciae anno $40 \ldots$ D. Wolfgango Thalhusero XL fl», UAT 6/7a, f. $207 \mathrm{v}$.

25 Vgl. Acta Senatus UAT II 1 a, f. 232 v-234r, außerdem Brief von Herzog Ulrich an die Universität, unterzeichnet vom Erbmarschall Hans Conrad Thumb, vom 5.August 1540 (UAT 14/1 f, 4r und v), Konzept des Antwortschreibens von Rektor und Regenten vom 6. August 1540 (UAT 14/1, f. 6r-7v). 
ein Schützling Melanchthons, war als Magister nach Tübingen gekommen, hatte dort Ethik gelesen und nebenher Medizin studiert und war im Jahr 1539 zum Doktor der Medizin promoviert worden. Aber Hawenreut(t)er mußte gegenüber Thalhäuser resignieren, er erhielt durch Vermittlung von Camerarius einen Ruf an das Gymnasium von Straßburg, wo er der Vorgänger von Winther von Andernach war ${ }^{26}$. Daß die Sympathien von Fuchs bei Hawenreut(t)er waren, können wir indirekt daraus schließen, daß er weiter mit ihm in Verbindung blieb ${ }^{27}$. Thalhäuser dagegen ist nach wenigen Jahren recht klanglos aus dem Kollegium verschwunden ${ }^{28}$. Fuchs würdigt ihn in den Briefen keiner Erwähnung.

Von den späteren Kollegen taucht nur Jakob Degen, genannt Scheck, auf. Fuchs lobt, er sei einer der wenigen, die sich nicht dem Interim gebeugt hätten ${ }^{29}$.

Die Episode Thalhäuser macht einen der weiteren Gründe für das Ùnbehagen von Fuchs in Tübingen deutlich: es sind die Differenzen mit den Hof beamten. Fuchs war wohl der Meinung, es werde zuviel von außen her und mit Unverständnis in die Belange der Hochschule eingegriffen, und er machte seinem Unmut gegenüber den Hofleuten deutlich Luft, so daß sich Camerarius zeitweise um ihn sorgte, denn er hatte die gefährlichen Anfänge der Auseinandersetzung in Tübingen noch miterlebt ${ }^{30}$. Aber Fuchs verschaffte sich damit im Gegenteil Respekt. Es scheinen auch konfessionelle

26 Vgl. Friedrich Wieger, Geschichte der Medizin und ihrer Lehranstalten in Straßburg vom Jahre 1497 bis zum Jahre 1872, Straßburg 1885, S. $32 \mathrm{f}$.

27 «Argentoracensium res, ut mihi paucos ante dies D. Sebaldus per literas significavit, ita habent» (Brief 14 vom 19. Februar 1549). Den erwähnten Brief gab Fuchs Johannes Brenz zu lesen, denn dieser schrieb am 5.März 1549 an Vitus Theodor: «Postquam has literas scripsi, legi doctoris Sebaldi medici literas ex Argentorato ad quendam amicum». Zit. nach Th. Pressed, Anecdota Brentiana, Tübingen 1868, S. 298.

${ }^{28}$ Vgl. die Eintragung in den Jahresrechnungen des Supremus Deputatus: «Item alls Doctor Wolf Thalheuser wollt hinweg, hat Ime die Universität decerniert XXX gulden » (UAT 6/7b, f. 59r (1544/45).

29 «D. Christophorus [Wiest] cum universa familia ante duos menses Coburgum est profectus, ut nunc ego cum Scheckio et Benigno solus relictus sim, qui se $\tau \tilde{\omega} \mu \varepsilon \tau \alpha \xi \dot{v}$ non contaminarit» (Brief 12 vom 13. Dezember 1548).

30 "Quod tu et Zieglerus meo dialogo estis perturbati et coniecti in metum de me, nihil miror. Ad ea enim quae haud ita dudum praeterierunt exempla respexistis. Sed nunc alia ratio est, atque adeo quosdam suorum consiliorum satis sero poenitet. Videntur iam post acerbam istam expostulationem me maiori quam antea nunquam complecti favore. Et vocant me non raro ad convivia sua, atque mecum colloquuntur interdum de scholae rebus» (Brief 2 vom 15. Juni 1542). 
Gegensätze eine Rolle gespielt zu haben. Für Fuchs als überzeugten Lutheraner hatten die «Zwinglianer» am Hof zu großen Einfluß. Er meinte damit wohl in erster Linie den Marschall Hans Konrad Thumb von Neuburg und konnte deshalb Schadenfreude kaum unterdrücken, als dieser und sein Bruder Hans Friedrich Thumb von Neuburg, der Obervogt in Kirchheim war, im Jahre 1543 aus ihren Ämtern gejagt wurden ${ }^{31}$. Herzog Ulrichs Rückkehr zu lutherischer Gesinnung glaubt Fuchs, was uns makaber anmutet, dadurch bewiesen zu sehen, daß der Herzog ein strenges Edikt gegen alle Juden erließ, nachdem er «im Bade» (Wildbad ?) Luthers Pamphlet gegen die Juden gelesen hatte ${ }^{32}$. Obwohl nun die Zwinglianer am Hofe «mit gesenkten Häuptern einhergingen», wie sich Fuchs ausdrückt, scheint sich sein Verhältnis zum Hofe auch in den folgenden Jahren nicht sehr gebessert zu haben. Er vermerkt zwar 1546 stolz, mehrmals zu Herzog Ulrich als beratender Arzt wegen dessen Podagra gerufen worden zu sein, möchte aber doch auf keinen Fall eine dauernde Stellung am Hof bekleiden ${ }^{33}$. Erst unter Herzog Christoph scheint man mehr auf seine Stimme gehört zu haben ${ }^{34}$. Bei ihm war er auch zeitweise Leibarzt ${ }^{35}$.

Schließlich war Fuchs - wiederum ein Grund des Verdrusses - mit der materiellen Versorgung in Tübingen nicht zufrieden ${ }^{36}$. Dabei gehörte er nach kurzer Zeit zu den am besten bezahlten Professoren der Universität.

31 Brief 4 vom 20. April 1543 und Brief 6 vom 11. Juni 1543.

32 «Hoc verissimum esse scio principem nostrum propter lectum sibi in thermis Lutheri adversus Iudaeos libellum confestim e ditione sua exturbasse et severissimo edicto proscripsisse omnes Iudaeos. Ita ut nunc fere melius sperare incipiam de rebus nostris,

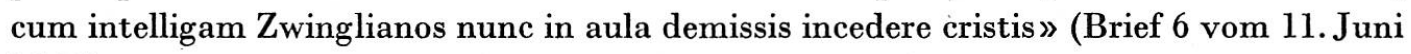
1543).

Ein solcher Erlaß ist nicht bekannt. Im HStASt konnten sich keine Unterlagen dazu nachweisen lassen.

33 «Tertio enim intra quatuordecim dierum spatium ad principem nostrum sum vocatus, quem podagra exercet. Medicum cuius antea usus est opera, propter facinus non admodum probandum, coniecit in carceres. Liberatum tamen iri spes est») (Brief 9 vom 1. März 1546). Der in den Kerker geworfene Leibarzt war Georg Kölblin, vgl. seine Urfehde vom 8. März 1546 (HStASt A 20 Urk. 16) und die Kassation seiner Urfehde vom 20. Juli 1555 (HStASt A 20 Urk. 15) s. a. Neues Württembergisches Dienerbuch, bearbeitet von Walther Pfeilsticker, Band 1, Stuttgart 1957, § 328.

${ }^{34}$ Vgl. den in Anm. 3 erwähnten Briefwechsel mit Herzog Christoph.

${ }^{35}$ Vgl. Neues Württembergisches Dienerbuch, bearbeitet von Walther Pfeilsticker, Band 1, Stuttgart 1957, § 331.

36 «Hic, ut nosti, praeter salarium nihil lucri accedit, quanta enim nostrorum sit rusticitas et inopia probe etiam perspectum habes» (Brief 8 vom 1. November 1544). 
Schon seit 1537 bekam er ein jährliches Gehalt von 200 Gulden, dazu einen «Hauszins» (ein Wohnungsgeld) von 14-15 und später 20 Gulden ${ }^{37}$. Hinzu kamen Nebeneinnahmen aus Dekanats- und Rektoratsführung und sicherlich auch aus ärztlicher Praxis, ganz abgesehen von den Naturaliengaben der Universität, die nur teilweise mit dem Gehalt verrechnet wurden. Aber nicht einmal zu der Zeit, als Fuchs sowohl 200 Gulden für seine Professur als auch 200 Gulden für seine Tätigkeit als Leibarzt bei Herzog Christoph bekam, scheint er zufrieden gewesen zu sein. Herzog Christoph mußte sich 1553 beschweren, weil Fuchs nicht ohne eine zusätzliche Bezahlung die Hebammen und Wundärzte in Tübingen examinieren wollte ${ }^{38}$. Nach Herzog Christophs Meinung gehörten solche Prüfungen seit jeher zu den Pflichten der Professoren in Tübingen.

Freilich hatte Fuchs eine große Familie zu versorgen. Und er wollte gern, daß seine zehn Kinder (vier Söhne und sechs Töchter) standesgemäß aufgezogen würden. Doch nur der älteste Sohn, Friedrich, scheint die Idealvorstellungen des Vaters erreicht zu haben: nach dem Studium der Medizin in Tübingen und der Promotion in Padua bekam er eine angesehene Stadtarztstelle in Ulm und heiratete, wie einst sein Vater, ein Mädchen aus einem Ratsherrengeschlecht ${ }^{39}$. Mit den anderen Kindern scheint Fuchs manchen Kummer gehabt zu haben. Seine beiden Söhne Daniel und David hielt er für verschwenderisch, so daß er für die Zeit nach seinem Tode Vormünder für sie einsetzte ${ }^{40}$. Immerhin studierten beide, doch nur Daniel brachte das Studium zu Ende (auch er wurde Mediziner), während David zum Stadtschreiber nach Ulm kam ${ }^{41}$. Salomon dagegen, der Jüngste, ein «filius perelegans », wie der Vater bei der Geburt 1546 stolz schrieb ${ }^{42}$, dachte nie ans Studieren und zog, nachdem er ein Handwerk erlernt, unstet umher ${ }^{43}$. Er

37 Vgl. die Eintragungen in den Rechnungsbüchern UAT 6/7a, f. 72v, 6/7b,f. 21 v und $56 \mathrm{r}$ usw., $6 / 7 \mathrm{c}, \mathrm{f} .143 \mathrm{r}$.

38 Vgl. das Konzept des Schreibens von Herzog Christoph an Fuchs vom 6. Juli 1553 (HStASt A 274 B. 63).

39 «Fridericus medicinae doctor Ulmae moratur publico stipendio a senatu urbis conductus. Duxit uxorem e genere patricio» (Brief 22 vom 22. Mai 1559).

40 Vgl. R[einhold] RaU, Zur Lebensgeschichte des Dr. Leonhard Fuchs und seiner Familie, in Tübinger Blätter 38 (1951) 44 f. - Die Pflegschaftsakten für die Söhne Fuchs befinden sich im UAT 44/125 X (1566-1572).

41 «David Ulmae apud scribam agit» (Brief 22 vom 20. Mai 1559).

42 «Peperit mihi nuper uxor filium perelegantem ...» (Brief 9 vom 1.März 1546).

43 «Unus Salomon, qui perpetuo animum a studiis alienum habuit, mechanicam artem edoctus, hinc inde vagatur» (Brief 22 vom 20. Mai 1559). 
starb schon 1562, ebenso wie Fuchsens jüngste Tochter Rebekka und deren Mann, der Apotheker Michael Weigelin, während einer Seuche in Bayern ${ }^{44}$.

Epidemien haben Fuchs auch das Leben in Tübingen verleidet. Er hielt Tübingen für ein besonders ungesundes Pflaster. «Du weißt ja, wie langsam die Seuche in Tübingen umgeht und daß sie nicht leicht vor eineinhalb Jahren aufhört », schrieb Fuchs 1555 an Camerarius ${ }^{45}$. Immerhin hat er in Tübingen mindestens zwei große Epidemien erlebt, vielleicht auch noch den Beginn einer dritten in seinem Todesjahr 1566. Die Seuchen in den Jahren 1541-1543 und 1554-1556 brachten der Universität schwere Einbußen, da sich Lehrer und Studenten zerstreuten ${ }^{46}$. Auch Fuchs meinte, ganz der traditionellen Anschauung verhaftet, das einzige Mittel gegen die «contagia pestis » sei die Flucht, und er reagierte als Rektor der Universität bei der Epidemie 1554/55 recht ärgerlich, als die Regierung nicht rasch und eindeutig genug in die Evakuierung der Universität einwilligte ${ }^{47}$. Er selbst floh mit seiner Familie während dieser Epidemien einmal nach Rottenburg, dann nach Herrenberg. Er als der berühmte Professor wurde dann gern von dortigen Patienten konsultiert ${ }^{48}$. Aber von seiner Arztpraxis spiegelt sich kaum etwas in seinen Briefen, und was wir hören, bleibt ganz im Rahmen des Üblichen. Einmal fragt er, ob Camerarius die Guajak-Kur, die er ihm wohl empfohlen hatte, geholfen habe ${ }^{49}$. Ein andermal klagt er, er

44 «Mense prioris anni octobre in Bavaria ex peste perierunt gener meus, filia Rebecca, et filius Salomon» (Brief 24 vom 3. April 1563).

45 «Nosti enim quam lente pestis Tubingae grassetur, nec facile ante annum unum dimidio desinat. Haec certe scholae nostrae, quae tum erat florentissima, maximum et propemodum insanabile inflixit vulnus» (Brief 20 vom 26. Februar 1555).

46 Zur Epidemie von 1541 bis 1543 vgl. Brief 2 vom 15. Juni 1542 und Brief 3 vom 23. November 1542, die beide in Rottenburg geschrieben wurden, außerdem den Eintrag von Fuchsens Hand im Fakultätsrechnungsbuch (Codex accepti et expensi) UAT 14/18, f. 6 v. Zur Epidemie von 1554 bis 1556 vgl. Brief 20 vom 26. Februar 1555 aus Herrenberg, außerdem die Briefe des Hofes vom 31. Dezember 1554 (UAT 6/1, f. 25 r), ebenfalls vom 31. Dezember 1554 (UAT 6/1, f. 27r und v.), vom 5. Januar 1555 (UAT 6/1, f. $30 \mathrm{r}$ ) und vom 12.Juni 1555 (UAT 6/1, f. 32r), außerdem den undatierten Brief, den Fuchs als Rektor an den Senat der Universität schrieb, vermutlich in Herrenberg in den ersten Januartagen 1555 (UAT 6/1, f. 28r).

47 Vgl. den in Anm. 46 erwähnten Brief von Fuchs UAT 6/1, f. 28 r.

48 «Ego qui hoc tempore taedia tot migrationum devorare cogor, et nunc etiam tanquam novus hospes ad aegrotos crebro vocor plura scribere non potui» (Brief 2 aus Rottenburg vom 15. Juni 1542).

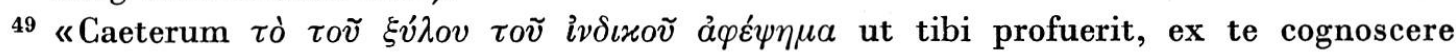
cupio» (Brief 2 vom 15. Juni 1542). 
werde von den Bauern mit Bitten um Urinschau überhäuft ${ }^{50}$. Von der Beratung Herzog Ulrichs ${ }^{51}$ und der Leibarzttätigkeit bei Herzog Christoph erfahren wir nichts Näheres. Insgesamt scheint die Praxis je länger je weniger eine Rolle im Leben von Fuchs gespielt zu haben.

Unruhe brachte für Fuchs vor allem die Beteiligung am reformatorischen und das Betroffensein vom politischen Geschehen. Fuchs war mit der theologischen Problematik seiner Zeit wohl vertraut, pflegte ständigen Umgang mit führenden Köpfen der Reformation (hier ist besonders Melanchthon zu nennen ${ }^{52}$ ) und stand vor allem in engem Kontakt mit Johannes Brenz ${ }^{53}$, dem Reformator Württembergs. Brenz scheint seine theologische Haltung entscheidend bestimmt zu haben: die kompromißlose Ablehnung des Interims ebenso wie die vermittelnde und vorsichtige Beurteilung des Osiandrischen Streites.

Nach dem für die Protestanten schmählichen Ausgang des Schmalkaldischen Krieges hatte Karl V. 1548 das Interim erlassen, jenes Einheitsgesetz für Katholiken und Protestanten, das auf Überbrückung der konfessionellen Gegensätze zielte, in der Praxis aber zum Ausnahmegesetz für die Protestanten wurde, das mit der bloßen Konzession von Laienkelch und Priesterehe in Lehre und Kultus Rekatholisierung bedeutete. Fuchs sieht klar, daß sich Herzog Ulrich aus politischen Gründen nicht der Verkündigung des Interims in Württemberg widersetzen kann ${ }^{54}$, aber er ist entsetzt über den Opportunismus der Tübinger Professoren ${ }^{55}$. Nach der

50 «Avocatus autem ter sum per rusticos quosdam, qui me hodie praeter consuetudinem lotiis suis obruerunt» (Brief 1 von 1541 ?).

$51 \mathrm{Vgl}$. die in Anm. 33 zitierte Briefstelle.

52 Melanchthon wird in den Briefen 2, 3, 4, 5, 8, 11, 12, 13, 15, 17, 18, 21 und 22 erwähnt.

${ }_{53}$ Brenz wird in den Briefen 5, 11, 15, 16, 17, 18 und 25 genannt. Fuchs ist z. B. auch über das Versteck von Brenz im Jahre 1551 orientiert: «Concessit in id monasterium, quod tu nosti [Sindelfingen] ... Ego vehementer metuo insidias, nam monasterium illud extra oppiduli moenia situm est, neque frustra » (Brief 15 vom 4. Juni 1551).

54 «De religione, quamquam $\tau \grave{o} \mu \varepsilon \tau \alpha \xi \dot{v}$ coactus princeps noster accepit, nihil adhuc est immutatum ...» (Brief 11 vom 7.August 1548). Zur Situation in Württemberg vgl. Gustav Bossert, Das Interim in Württemberg, Halle 1895 (= Schriften des Vereins für Reformationsgeschichte 46/47).

55 «Schola nostra non nisi ex pessimorum hominum fece et sanguinariis papistis concinnata mirifice nunc triumphat, et virus suum ex intimis visceribus eructat. Nemo tamen magis quam Sichardus et scriba ille, quem tu semper, monitus etiam ab amicis, in oculis tamen tulisti, fraudibus ac dolis consutum hominem » (Brief 11 vom 7.August 1548). Mit «Scriba ille» könnte der ehemalige Stadtschreiber (bis 1538) Caspar Volland gemeint sein. 
Vertreibung des Reformatos Erhard Schnepf(f) aus Tübingen seien Scheck, Benignus und er die einzigen, die sich nicht mit dem Interim befleckt hätten, schreibt er schon am 13. Dezember 1548 an Camerarius ${ }^{56}$. Nicht zufällig ist Fuchs 1549 Nachfolger von Schnepf(f) als Superattendent des Theologischen Stifts in Tübingen geworden ${ }^{57}$.

Beim Osiandrischen Streit, einer innerlutherischen Auseinandersetzung in den Jahren nach 1549, geht es um das Zentrum lutherischen Glaubens: die Rechtfertigungslehre. Osiander sah gegenüber dem (vor allem von Melanchthon vertretenen) forensischen Verständnis der Rechtfertigung als Gerechterklärung auch ihre verändernde Kraft, verstand sie als Gerechtmachung, als reale Einwohnung der wesentlichen Gerechtigkeit Christi im Gläubigen ${ }^{58}$. Osiander machte mit seinem heftigen Temperament seinem Namen in dem Streit keine Ehre. Fuchs aber fand, man dürfe dem « wilden Mann» («agrion andra», wie er im Wortspiel zu Osiander = frommer Mann sagt) auf der Gegenseite nicht mit der gleichen Heftigkeit begegnen ${ }^{59}$. Im Bemühen um Vermittlung meinte er wie Brenz, im Grunde handle es sich um ein bellum grammaticale ${ }^{60}$. Ganz so Unrecht hatte er damit nicht, denn auch die Orthodoxen konnten mit ihrem Rechtfertigungsverständnis nicht gut sittliche Laxheit und untätiges Christentum begünstigen wollen.

Fuchs zeigt sich immer wieder bemerkenswert gut informiert, ob es nun um den Verlauf jenes erfolglosen Regensburger Religionsgespräches von 1546 geht $^{61}$ oder um jene Synode 1551 in Stuttgart, auf der die Confessio 56 Vgl. Anm. 29.

57 Vgl. den Eintrag im Statutenbuch des Theologischen Stifts Tübingen (beglaubigte Abschrift) S. 231: «Abiit [sc. Erhard Schnepf] ad angariam Divi Georgii anno 1549, in cuius locum statim successit D. Leonhardus Fuchs» (Archiv des Theologischen Stifts Tübingen, Kasten II, F. 12, Nr. 1).

58 Vgl. E. Brzer, Art. Andreas Osiander, in Religion in Geschichte und Gegenwart, 3. Auflage, Band 4 (1960) Sp. $1730 \mathrm{f}$.

59 «Neque nunc placet, ut vehementius aliquid adversus $\tau \dot{\nu} \nu a ̆ \gamma \varrho \iota \nu \nu ~ a ̆ v \delta \varrho \alpha$ a vestris geratur. Nec huius meae sententiae alias affero causas, quam quod antea abunde satis res nostrae afflictae sint » (Brief 16 vom 3. September 1551). Weitere Stellungnahmen zum Osiandrischen Streit siehe Briefe 15, 17 und 18.

60 «Non probatum tibi Brentii conciliatio, et putas hac ipsa bonis et piis multum tristitiae allatum esse, et improborum ac turbulentorum $\dot{\pi} \iota \chi \varepsilon \iota \eta ́ \sigma \varepsilon \iota \varsigma$ fulcitas. Imo ego multum gaudii piis peperisse arbitror, ut qui nunc intelligant hoc bellum quod utrinque summa contentione, nec citra convitia, gestum est, grammaticum potius quam theologicum fuisse» (Brief 18 vom 23. April 1553).

61 «Schnepphius adhuc Ratisponae haeret, sed nihil, quod sciam, tribus iis mensibus quibus abest actum est, nisi quod Hispanus monachus [sc. Petrus Malvenda] monstrosas quasdam propositiones de iustificatione illis obtrusit ...» (Brief 9 vom 1. März 1546). 
Wirtembergica geprüft und gebilligt wurde ${ }^{62}$. Das gilt auch für die politischen Ereignisse. Wir sahen es schon in bezug auf die innerwürttembergischen Verhältnisse (Aff äre Thumb u. ä.), aber es zeigt sich auch beim Zwist in Sachsen ${ }^{63}$, beim Türkenkrieg ${ }^{64}$, bei den Nachrichten über den Abstecher von Kaiser Karl V. nach Stuttgart ${ }^{65}$ im Jahre 1543 sowie über den Schmalkaldischen Krieg und seine Folgen ${ }^{66}$. Fuchs floh bei der Besetzung Württembergs durch das fremde Militär im Jahre 1547 nach Rottweil, kehrte aber schon nach etwa einem Monat zurück ${ }^{67}$. Mit gespanntem Interesse verfolgte Fuchs natürlich auch jenen Überfall des «deutschen Michels» auf Ellwangen im Jahre 155268. Deutscher Michel - das ist der Spitzname des Deutschmeisters Wolfgang Schutzbar genannt Milchling.

Bei soviel äußerer und innerer Unruhe ist es um so bewundernswerter, mit welcher Stetigkeit Fuchs sein wissenschaftliches Werk vorantrieb, wie er keine Möglichkeit ungenutzt ließ, um wissenschaftliche Verbindungen zu knüpfen und zu erhalten, etwa nach Paris zu Jacob Goupyl, um wertvolle

62 «Nostri nunc Stugardiae conventum habent, deliberaturi de concilii rebus, ut arbitror» (Brief 15 vom 4. Juni 1551).

${ }^{63}$ «Priores [sc. literae] turbas apud vos excitatas habuerunt, quas Lantgravius pro sua prudentia probe composuit.» (Brief 2 vom 15. Juni 1542).

64 «Finito nunc tandem, nec admodum feliciter, Turcico bello, et domum reverso Electore Marchione ...» (Brief 3 vom 23. November 1542).

65 «Caesar Stugardiam ad principem nostrum divertit [22. Juli 1543], ubique noctem unam moratus iterum abiit. Petiit a principe, ut illi in publico loco missam papisticam instituere per suum sacrificum liceret. At princeps rogavit, ne tale aliquid ab eo petat, quod bona conscientia praestare non possit. Permisit itaque, ut intra parietes suae habitationis missam haberet » (Brief 7 vom 7. August 1543).

${ }^{66}$ «Primum autem scias me meamque familiam, Dei gratia, perbelle valere, neque quicquam iacturae nos per universum hoc belli tempus sane turbulentissimum in hunc usque diem fecisse, nisi quod anno ab hinc propemodum altero quum Rotvilam confugeremus, maiores sumptus fecerimus. Vix autem mensem illic morati, Tubingam reversi sumus. Hoc anno in maximo fuimus metu, ne intra moenia nostra se reciperet Hispanus et peregrinus miles, nam passim vicina loca occupaverat. Quare aliquam partem mearum fortunarum alio transferre sum coactus, ne ex inopinato nos obrueret. Proxima septimana in unum omnes locum praeter omnium expectationem concesserunt. Ascenderunt autem per eam vallem, quae ducit Ballingam et Rotvilam, ubi in hunc diem commorari dicuntur. De illorum instituto quod scribam certi nihil habeo. Varii enim sparguntur rumores, conveniunt tamen de bello Helvetiis inferendo » (Brief 11 vom 7. August 1548).

${ }^{67}$ Vgl. Anm. 66.

68 «Tamen est quod de bello nostro ad te scribam, quod a Teutonico illo Michaele - sic enim passim apud nos vocatur - est excitatum, ob occupatam per insidias, et clam nostro principe, Elvangam, quae hactenus illo tutore ac patrono usa est » (Brief 17 vom 14.Dezember 1552). 
Handschriften antiker medizinischer Autoren kennenzulernen ${ }^{69}$, oder auch zu anderen in der Botanik besonders interessierten Männern wie Johannes Heß oder Hieronymus Herold in Nürnberg ${ }^{70}$. Die interessantesten Nachrichten sind die über Entstehung und Wachstum der Historia stirpium.

Unser Briefwechsel beginnt ja mit einer botanischen Diskussion, die zur 1542 erschienenen Ausgabe der Historia stirpium gehört. Wir bekommen Einblick in das Dedikationsgebaren der Zeit (Fuchs hatte diese Ausgabe dem Kurfürsten Joachim II. von Brandenburg gewidmet und sandte einen Boten mit dem fertiggestellten Buch zu ihm, wobei er um Vermittlung und Empfehlung durch Melanchthon und dessen Schwiegersohn, den Humanisten Georg Sabinus bat) ${ }^{71}$, und wir hören von dem hohen Preis, den man für das Werk bezahlen mußte: nicht weniger als 15 Gulden ${ }^{72}$.

69 «Nicolaum $\mu v \varrho \varepsilon \psi \iota x \dot{v} v$ nunc propria manu describo, quod graece quoque illum edere constituerem. Confirmat hanc sententiam Iacobus Goupylus Gallus vir doctissimus, quique nobis Trallianum emendatissimum exhibuit, quod nuper ad me ex Parisiis scribens pollicitus nobis sit prolixe suam et totius sui collegii operam. Nam sunt illic tria Hippocratis graeci exemplaria venerandae antiquitatis, quorum unum ad marginem Herodiani grammatici scholia adscripta habet, quibus ea quae ad dialecton ionicum pertinent explicat. Et brevis quaedam Lyci $\grave{\varepsilon} \xi \dot{\eta} \gamma \eta \sigma \iota \varsigma$ multorum locorum obscurorum. Galeni multi commentarii manu descripti custodiuntur religiosissime. Sunt Lutetiae Aetii 4 exemplaria graeca. Totidem Alexandri Tralliani. Totidem item Oribasii, Pauli, Actuarii, Nicolai Myrepsi. Quae te quoque scire volui, ut esset quo te oblectares, et cognosceres bonam spem fore, ut brevi optimos quosque autores graecos simus habituri» Brief 10 (eigentlich Postskript zu Brief 9 vom 1.März 1546).

70 «Caeterum a te maiorem in modum peto, ne graveris nomine meo d. D. Ioannem Hessum reverenter et amanter salutare eumque monere, ut aliquando stet promissis, et mihi stirpes vel earundem icones mittat. In primis autem Ambrosiam virentem, aut si id illi integrum non fuerit, saltem iconem primo quoque tempore ad me transmittat. Facies etiam mihi gratissimum, si clarissimum virum D. d. [sic] Hieronymum Heroldum rei herbariae diligentissimum inquisitorem, accurata diligentia mea salute impartire haud gravatus fueris» (Brief 27 an J. Camerarius d. J. vom 18. April 1564).

${ }^{71}$ «Finito nunc tandem, nec admodum feliciter, Turcico bello, et domum reverso Electore Marchione, commentarios meos quos de stirpium historia inscripsi per hunc adolescentem illuc transmittere decrevi. Quod institutum meum ut pro virili tua iuves, te per amicitiam nostram obtestor.... Iuvabis autem mea quidem sententia plurimum, si initio ad Philippum nostrum [sc. Melanchthonem] perscripseris, measque nugas ut principi commendare non dedignetur, illi studiose persuaseris. ... Dein si ad Sabinum aut alium literas dederis, cuius opera adolescenti prodesse in aula, ut ad principem cum codice aditum habeat facilem, possit» (Brief 3 vom 23. November 1542).

72 «Ut vero hoc quale sit perspectum habeas, adolescenti huic demandavi, ut codicem explicet tibique videndum exhibeat. Utcunque autem ornatus sit, nemo hunc ita in- 
Kaum daß 1543 die deutsche Ausgabe, das «New Kreüterbůch», erschienen war ${ }^{73}$, da arbeitet Fuchs schon (1544) am 2. Band, an der Fortsetzung und Erweiterung seiner Pflanzengeschichte ${ }^{74}$. Erst im Jahre 1555 kommt er zu einem vorläufigen Abschluß: «Meine Kommentare über die Pflanzengeschichte, eingeteilt in zwei Bände großen Gewichts, ein kostspieliges und mühevolles Werk, will ich Anfang kommenden Sommers in Druck geben. Sie werden mehr als tausend Beschreibungen und sehr schön wiedergegebene und gemalte Pflanzen enthalten. ${ }^{75}$

Aber es drängt Fuchs immer wieder zur Erweiterung. 1557 schreibt er, zur Entschuldigung seltenen Schreibens, er sei ganz mit der Vollendung der Pflanzengeschichte beschäftigt: «Ich habe zwei Bände vollendet, von denen jeder 400 Bilder hat. Ich bin jetzt beim 3. Band, der auch 400 Bilder enthalten soll, bis zum Kapitel 80 fortgeschritten. ${ }^{76}$ Natürlich möchte Fuchs seine Konkurrenten, die botanisch arbeiten, überflügeln, insbesondere Pietro Andrea Mattioli, jenen «stolzgeschwellten Italiener», den er nicht leiden kann ${ }^{77}$, aber auch Konrad Ges(s)Ner, der selbst an einer großen Pflanzengeschichte arbeitet und Fuchs in bester Absicht Zusammenarbeit vorschlägt, was dieser brüsk zurückweist ${ }^{78}$.

structum minoris quam quindecim florinis comparare posset.» (Brief 3 vom 23. November 1542).

${ }^{73}$ Leonhart Fuchs, New Kreüterbuch [etc], Basel 1543.

74 "Adorno iam alterum tomum de stirpium historia, in quo recentes et novae, quaeque in priore desiderantur, erunt herbae» (Brief 8 vom 1. November 1544).

75 «Commentarios meos de stirpium historia in duos magnae molis tomos digestos, opus sumptuosum et laboriosum, ad aestatis futurae initia praelo subiciam. Plusquam mille stirpium historias et imagines pulcherrime expressas et effigiatas habebunt » (Brief 20 vom 26. Februar 1555).

${ }^{76}$ «Et remorantur me non raro studia mea cur minus ad amicos scribere liceat, praesertim hoc tempore, quo totus sum in absolvendo eo opere, quod de stirpium historia meditor. Perfeci duos tomos, quorum singuli quatuor iconum centurias habent. Sum nunc in tertio progressus usque ad caput 80 , qui totidem habebit imaginum centurias. Mole sua prioris editionis opus si non superant, certe aequant. Spero hunc meum laborem posteritati profuturum. Historiae singulae erunt prolixiores et accuratiores quam prior editio, quae prae hac sordebit, habeat» (Brief 21 vom 16. Mai 1557).

77 "Qualia sint edita a Matthiolo intelligent plurimi, ubi, Deo adiuvante, prodierint commentarii mei. Quippe singulis horum tomis, nisi me fallit memoria, centies ille a me reprehensus est. Sentiet haud dubie fastu turgidus ille Italus, in Germania esse viros, qui nugas ipsius animadverterunt, et eum suis coloribus pinxerunt» (Brief 26 vom 24. November 1565).

78 "Quod Gesneri opus ingens in singulari apud quosdam sit expectatione, valde miror, quandoquidem ille ante annum fere ad me scripserit, se nedum sylvam eius operis sibi 
Im Jahre 1563 schreibt Fuchs, er habe seine Pflanzengeschichte längst fertiggestellt ${ }^{79}$. Sie soll nun in drei mächtigen Bänden mit über 1500 Bildern erscheinen. Aber der Witwe von Michael Isingrin, seinem alten Verleger, mit der bereits ein Vertrag abgeschlossen wurde, ist die Herausgabe des Werkes, die 3000 Gulden kosten soll, zu risikoreich. Die (offensichtlich weitgehend fertiggestellten) Druckstöcke mit den Abbildungen sollen an einen anderen Drucker verkauft werden ${ }^{80}$. Fuchs macht einen letzten Versuch und bittet alle möglichen Fürsten und Reichsstädte um Unterstützung bei dem Druck ${ }^{81}$. Aber noch ehe es zum ersehnten Ziel der Drucklegung kommt, stirbt Fuchs, der in den letzten Jahren durch Krankheit und Nachlassen der Sehkraft erheblich geplagt war ${ }^{82}$, im Jahre 1566.

adhuc parasse. Fac vero ante meum prodeat, non laboro, ut qui Gesneri, optimi sane amici, et crebro ad me scribentis, animum perspectum habeam, per omne fere genus autorum circumvolitantis, et ex retextis aliorum sertis, novas subinde corollas concinnare gaudentis. Ita nuper universum Rondeletii librum in suos de piscibus commentarios retulit. Pari ratione a me petiit, si ei permittam, ut pro arbitrio singula disponat, se post editos meos commentarios Paralipomena scripturum. Disponat ipse sua ut libet, ipse non patiar, ut mea secus ac ipse feci, disponat. Iam cupit a me de omnibus meis ut illum certum faciam petere. Sed sentio quid moliatur, ideo ei deinceps nihil mearum stirpium mittam» (Brief 26 vom 24. November 1565).

79 «Quod ad lucubrationes meas attinet, commentarios meos de stirpium historia in tres magnae molis tomos digestos iam pridem absolvi. Fefellit fidem mihi vidua Isingrinii et eius gener, non obstante quod illam chirographo suo obstrinxerunt, ita nusquam, mi Ioachime, tuta fides est » (Brief 24 vom 3. April 1563).

80 «Imprimis vero absolvi opus omnium laborisissimum ac sumptuosissimum de stirpium historia, in tres maximae molis tomos digestum, icones continens mille quingentos et quatuor. De eius operis editione ante biennium convenit inter me et viduam Michaelis Isingrinii, cuius ea de re syngrapham habeo. Sed tanta est hominum perfidia, ut iam a promisso impudentissime resiliat. Tabulas vult vendere, iusto pretio, alteri typographo cui eius operis editio adluberet. Verum hactenus mihi nullus est indicatus» (Brief 27 an J. Camerarius d. J. vom 18. April 1564).

81 "Quum vero praeter sumptus, quos feci hactenus maximos, iam nemo typographorum sit, qui tantos quos eius operis editio postulat sumptus facere velit aut possit, nempe trium milium florenorum, nec ipse sine meorum liberorum, ut nosti, extrema iactura, tantum pecuniae suppeditare possim, id capere consilii compulsus sum, ut a summis principibus et imperialibus civitatibus per literas subsidia petam, ut illorum in me, aut potius medicinae et herbariae rei studiosos, munificentia tantum pecuniae consequerer, ut saltem aliqua ex parte typographo succurrere queam, et in publicum tandem hoc opus prodeat» (Brief 25 vom 10. August 1565).

82 Zur Krankheit vgl. Brief 24 vom 3.April 1563: «Anno ab hinc uno Thermis Ferinis usus sum. Sed paulo post in febrem incidi tertianam puram [statt puras!], quae me sic afflixit, ut plerique putarent me ex illa certo moriturum. Tandem vero, adiuvante Christo, 
Wo sind die Druckstöcke geblieben? Dem Schicksal jener 185 Tafeln mit Vorzeichnungen für den Holzschnitt, die einst in Tübingen aufbewahrt wurden und erst in unserem Jahrhundert zum größten Teil zerstört wurden, ist Stübler nachgegangen ${ }^{83}$. Heute sind noch 23 Tafeln vorhanden ${ }^{84}$. Aber es müssen weit mehr Tafeln fertiggestellt gewesen sein. Beweis dafür sind die im Platter-Herbar befindlichen Probedrucke von Fuchs-Holzschnitten ${ }^{85}$. Und noch im 18. Jahrhundert waren neben neuen Holzschnitten sämtliche Druckstöcke der ersten Ausgabe im Besitz von Johannes Ges(s)ner, dem Freund Albrecht von Hallers in Zürich ${ }^{86}$. Es wäre verwunderlich, wenn sich davon nichts erhalten hätte. Sie mögen unerkannt in irgendeinem Winkel schlummern.

convalui. Quanquam post unam et item alteram septimanam iterum me invasit, non tamen nisi terna accessione mihi molesta fuit.»

Zum Augenleiden vgl. Brief 22 vom 20. Mai 1559: «Valeo, Deo gratiae, satis mediocriter, atqui oculi iam aliquot annis caligine laborant. » Und Brief 23 vom 21. Dezember 1561: «Constitui mecum, quum oculi prorsus caligent, me asserere in otium.»

Vgl. außerdem den Eintrag in den Acta Senatus (UAT 2/1b, f. 182r) vom 2. November 1554: «Rector magnificus [Fuchs] petiit veniam a senatu, dieweil er an einem Auge verlötzt, daß er möge die lectiones ein Weil biß zur besserung suspendirn. Ist seiner Magnificentiae vergönt. » Sowie die Briefe von Fuchs an Rektor und Regenten der Universität Tübingen, die sich im UAT $14 / 1$ auf $\mathrm{f}$. $12 \mathrm{r}$ und $\mathrm{f}, 10 \mathrm{r}$ und $\mathrm{v}$ finden und die um Entlastung von der Vorlesungstätigkeit bitten. Der erste Brief stammt vermutlich aus dem Jahre 1562, der zweite aus dem Jahr 1563. Letzterer trägt einen alten, aber falschen Registraturvermerk, der ihn dem Jahr 1548 zuweist.

83 StüBler (1928), S. 101 (265) Anm. 2.

${ }^{84}$ Seit Stüblers Nachforschungen sind also zwei weitere Platten abhanden gekommen.

85 Vgl. Walter Rytz, Das Herbarium Felix Platters, Ein Beitrag zur Geschichte der Botanik des XVI.Jahrhunderts, Verh. Naturforsch. Ges. Basel, Band 44, 1.Teil, 1933, besonders S. 126-206.

${ }^{86}$ Salomon Schinz (Anleitung zu der Pflanzenkenntniß und derselben nützlichen Anwendung, Zürich 1774) ließ 100 der Fuchs-Holzschnitte von den alten Stöcken drucken und von den Kindern seines Waisenhauses kolorieren. Er bot dem Publikum an, alle weiteren Holzschnitte der Historia stirpium auch noch abzudrucken, falls Interesse dafür bestehe. Diese Holzschnitte sowie 20 Holzschnitte der geplanten Neuausgabe und 100 Probedrucke derselben seien im Besitz von Johannes Geßner (S. 119).

Außerdem befindet sich in der Zentralbibliothek Zürich die Handschrift der Historia plantarum von JoHanN JACOB Scheuchzer (Ms Z VIII 22) in 8 Bänden, von denen der 7. Band verloren gegangen ist. In den erhaltenen Bänden befinden sich 455 extra angefertigte Abdrucke der Fuchs-Holzschnitte. Es bedarf noch eines genauen Vergleichs, um festzustellen, ob sich darunter auch Probedrucke von Holzschnitten für die Neuausgabe der Historia stirpium erhalten haben. 
Fuchs schrieb einst: «Ich hoffe, daß diese meine Arbeit der Nachwelt nützen wird. ${ }^{87}$ Diese Hoffnung hat sich so nicht erfüllt. Aber nachdem das kostbare Manuskript der Neubearbeitung des Kräuterbuches von Fuchsens Hand vor Jahren wieder zum Vorschein $\mathrm{kam}^{88}$, gelingt es vielleicht auch, noch weitere Reste der Tafeln zu finden und zu retten. Es wäre eine einzigartige Gelegenheit, Fortschritt und Bedeutung des Fuchsschen Werkes bis in die Druckherstellung hinein sichtbar zu machen.

87 Vgl. Anm. 76.

88 Vgl. Anm. 3. 